\title{
A Física das Cidades
}

\author{
Fabiano L. Ribeiro 무
}

Universidade Federal de Lavras, Departamento de Física, Lavras MG, Brasil. Email: fribeiro@ ufla.br https://doi.org/10.47235/rmu.v8i1.159

\section{Introdução}

A palavra "física" pode ser entendida por pelo menos duas formas. Primeiro, pela origem grega da palavra que significa natureza. Nesse sentido, quando dizemos que queremos entender a "física" de um fenômeno, quer dizer que queremos entender o que é, como e por que esse fenômeno se comporta de tal maneira. Ou seja, entender a natureza de alguma coisa - seja um fenômeno natural ou um conceito - significa que somos capazes de entender os mecanismos que regem esta coisa. A segunda forma da palavra física tem a ver com a disciplina/área do conhecimento; isto é, todas as ferramentas teóricas e instrumentais desenvolvidas pelos físicos para tentar entender o universo em que vivemos. Esses dois sentidos da palavra "física" são, em certo sentido, apropriados para descrever uma vertente bastante atuante nos últimos anos para se entender $o$ fenômeno urbano: a busca pela natureza das cidades por meio de ferramentas teóricas provenientes da física.

Estamos vivendo um momento crítico na história humana. No ano de 2015 a população urbana mundial superou a população rural pela primeira vez. Só para se ter uma ideia, no final do século XIX mais de $80 \%$ da população vivia em áreas rurais. Hoje já temos $54 \%$ da população vivendo nas cidades e os especialistas concordam que até 2050 mais de $70 \%$ da população mundial viverá nas áreas urbanas. ${ }^{1}$ Dessa forma, é esperado que a grande maioria das pessoas venham a viver em cidades superpopulosas em um futuro próximo. A questão que surge então é: em que condições viveremos nessas cidades do futuro? A resposta a essa pergunta está intimamente relacionada com o que tem sido chamado e definido por ciência das cidades (Batty, 2013). O desenvolvimento (ou não) dessa nova ciência será mandatório para se saber em que tipo de cidade viveremos: se em cidades superpopulosas e sem condições básicas; ou se em cidades ainda superpopulosas, mas com dignidade e qualidade de vida.
A Ciência das cidades seria um conjunto de teorias e evidências empíricas para explicar e prever fenômenos urbanos. No que diz respeito aos dados disponíveis, podemos dizer que estamos em um momento bastante interessante. Hoje podemos observar nossas cidades da mesma forma que os biólogos observaram pela primeira vez os tecidos biológicos no final do século XVI quando o microscópio foi inventado. Hoje quase todas as pessoas carregam um smartphone no bolso registrando com precisão e de forma automatizada suas posições geográficas e suas escolhas de compras. Uma vez que esses dados são gerados aos milhares por segundo, os cientistas podem investigar em tempo real padrões de mobilidade humana, tráfego de veículos nas ruas, transações financeiras, e assim por diante. Esse volume absurdo de dados oferece oportunidades sem precedentes que pode e deve ser explorado para melhorar a qualidade e a dinâmica das pessoas nas cidades.

Mas, é claro, apenas dispor dessa enorme quantidade de informação não significa entender o fenômeno urbano. Os dados nos permitem descrever alguns aspectos do comportamento urbano, porém, isso por si só não é suficiente para explicar a natureza (a física) das cidades. A natureza das cidades só pode ser alcançada (se puder ser alcançada!) se tivermos alguma teoria que explique $o$ fenômeno urbano, mesmo que de uma maneira muito simplificada. Aliás, o que entendemos por ciência é exatamente a interconexão entre dados e teoria. Os dados, por si só, não formam o que entendemos por ciência. Dados sozinhos são apenas uma coleção de números. A teoria por si só também não é ciência, mas apenas uma manifestação intelectual. A ciência surge de um ciclo ininterrupto que segue a ordem: dados fomentam teorias; teorias sugerem a coleta de novos dados; novos dados falseiam teorias e fomentam novas, e assim sucessivamente, como descrito na Figura 1. 


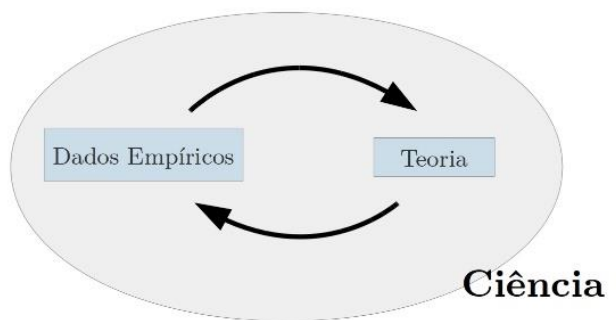

Figura 1. O que entendemos por ciência é um ciclo ininterrupto entre dados fomentando teorias; teorias sugerindo coleta de novos dados; e novos dados falseando e fomentando novas teorias (fonte: elaborada pelo autor).

Esse ciclo é feito em disciplinas como física, química, e biologia há pelo menos três séculos. Por exemplo, o conjunto de dados astronômicos que Tycho Brahe possuía na segunda metade do século XVI era apenas uma coleção de números. No entanto, quando esses dados foram descritos pela primeira vez por Kepler e posteriormente teorizados por Newton com sua Teoria da Gravitação, foi possível explicar como e porque os corpos se deslocam no espaço. As leis de Kepler e a Teoria Newtoniana, construídas a partir dos dados de Tycho Brahe (veja Figura 2), permitiram entender que as mesmas leis físicas que agem na queda de uma maçã também mantêm os planetas orbitando ao redor do sol.

Neste presente trabalho serão exploradas algumas ferramentas da física que têm contribuído para um melhor entendimento das cidades. Mais especificamente, será mostrado como propriedades de escala, como a dimensão fractal, podem revelar estruturas espaciais das cidades. Contudo, existem várias outras ferramentas que não serão exploradas aqui, mas que merecem total atenção. Por exemplo os conceitos de redes complexas, usados para entender as conexões entre pessoas, redes de ruas, redes de distribuições, etc. (Louf, Roth e Barthelemy, 2014; Haff, 2000; Asprone et al., 2013; Strano et al., 2012); modelos de sistemas dinâmicos e os modelos gravitacionais, usados para entender fluxos temporais e espaciais de produtos e pessoas inter e intra cidades (Bettencourt et al., 2007; Batty, 1971; Barthelemy, 2019; Birkon e Clark, 1991); modelos baseados em agentes e todo o conceito de ciência da complexidade, usados para entender propriedades macroscópicas a partir das interações locais e microscópicas (Mitchell, 2009; Boccara, 2004) ); bem como os conceitos de criticalidade auto-organizada (Batty, 2013 , Bak 1999; Ball 2004; Portugali 2000). Nesse sentido, o título deste artigo me parece oportuno, pois a palavra física traz os dois pontos que serão explorados aqui: 1) a apresentação de como alguns conceitos bem estabelecidos em física podem ser fundamentais para 2) entender a física (natureza) das cidades.

O artigo está organizado da seguinte forma. Nas seções (2) e (3) serão apresentadas algumas métricas urbanas que exibem propriedades fractais, i.e. que possuem propriedades similares em todas as escalas/tamanhos das cidades. Em especial, na seção (2), será apresentado o método boxcounting para a determinação da dimensão fractal de uma cidade. Na seção (3) será discutido como variáveis socioeconômicas e infraestruturais se relacionam com o tamanho das cidades. Na seção (4) será apresentado um panorama histórico do desenvolvimento da mecânica celeste e, em seguida, serão apresentadas analogias para identificar em que fase nos encontramos na construção da ciência das cidades. Finalmente, na seção (5) será discutido se as cidades podem realmente ser tratadas pelos ritos da ciência tradicional ou se seria necessário uma nova forma de ciência, baseada puramente nos dados, abandonando o conceito de teoria.

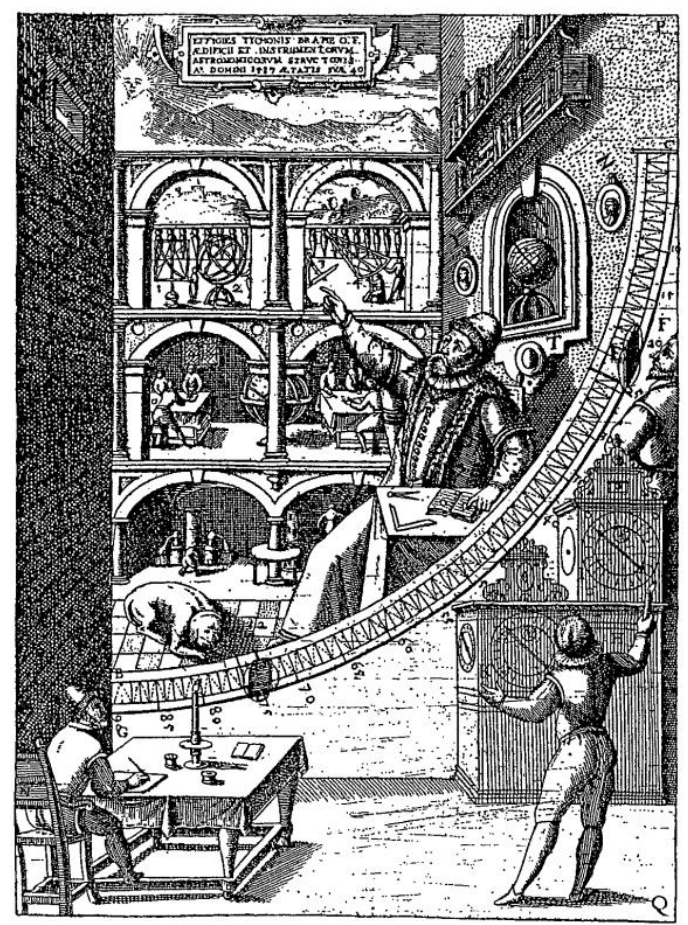

Figura 2. Ilustração do livro Astronomiae Instauratae Mechanica (1598), onde são 
apresentados os instrumentos de Tycho Brahe, que se encontra no centro da imagem (fonte: https://pt.wikipedia.org/wiki/Ficheiro:Tycho_Bra hes_stora_murkvadrant,_Nordisk_ Familjebok.jpg).

\section{Dimensão e Forma das Cidades}

A Figura 3 mostra a imagem do que se acredita ser a primeira cidade já construída pela humanidade, a vila/assentamento Çatal Hüyük (Whitfield, 2005). Ela foi erguida há cerca de 8.700 anos, onde hoje é a Turquia. $\mathrm{O}$ que é muito curioso sobre esta proto-cidade é que nela não há ruas ou caminhos conectando as casas. Provavelmente as pessoas acessavam as casas andando sobre os telhados. Chega ser difícil acreditar que essas pessoas pré-históricas não pensaram em caminhos para conectar as casas. No entanto, esse fato nos mostra que ter ruas conectando as casas não é, necessariamente, uma ideia óbvia.

Mas, é claro, posteriormente diferentes sociedades chegaram à ideia de introduzir caminhos e ruas para conectar as casas, e essa implementação mudou drasticamente a distribuição espacial das casas em comparação com o que vemos em Çatal Hüyük. Mas seria possível identificar algum tipo de padrão nessa distribuição espacial comum a uma boa parte de vilas ou cidades pelo mundo? Esse é um assunto importante em morfologia urbana e, com certeza, a física, como área do conhecimento, tem muito a oferecer e contribuir.

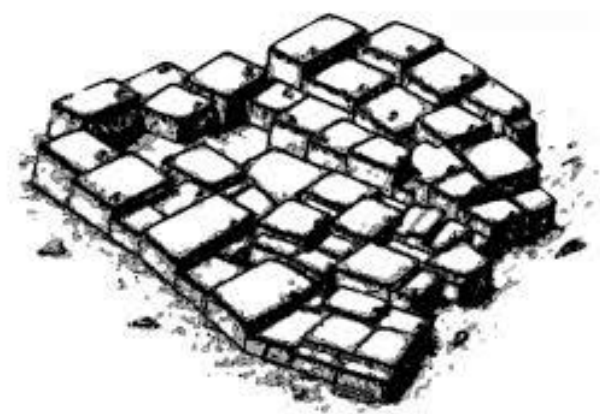

Figura 3. Imagem de Catal Hüyük, talvez a primeira cidade construída pela humanidade. Ela foi erguida há cerca de 10.000 anos onde hoje é a

Turquia. Nesta proto-cidade não há ruas ou caminhos conectando as casas. Provavelmente as pessoas acessavam as casas andando sobre os telhados (fonte:

https://upload.wikimedia.org/wikipedia/he/d/dc/H ouses_\%28reconstruction\%29_\%C3\%87atalh\%C 3\%B6y\%C3\%BCk2.jpg).
Um exemplo interessante de como a distribuição espacial das cidades acontece ou se forma vem de uma pequena vila do interior de Minas Gerais chamada Estação de Carrancas. Esta vila possui cerca de 200 habitantes e sua imagem é apresentada na Fig. (4). No início dos anos 70 a vila era formada por uma única rua (indicado pela seta na figura) construída em torno de uma ferrovia, tendo um mercado em uma das extremidades (ele ainda existe hoje). Este é um exemplo de cidade unidimensional, no sentido de que ela se estende ao longo de uma linha. De fato, a vila continuou sendo unidimensional até o começo dos anos 80, não invadindo uma segunda dimensão por causa da existência de uma colina ao lado da vila. Durante a década de 70, quando um cidadão decidia construir uma nova casa, ele tinha de escolher entre i) continuar seguindo a linha da rua e construir sua casa na última posição da linha; ou ii) construir a casa mais próxima do mercado, na colina; contudo, tendo que subir e descer esta colina todos os dias. Até o final dos anos 70 a primeira opção sempre vencia porque a distância entre a última casa e o mercado não era tão longa e caminhar até o mercado não era um problema. No entanto, no início dos anos 80, com um número suficiente de casas na linha e, consequentemente, uma maior distância do mercado, a segunda opção começa a ficar melhor. Neste momento, a vila invade a segunda dimensão espacial. Este é um exemplo de como a geografia (colina), economia e acesso à infraestrutura (mercado) interferem na distribuição espacial das casas e, consequentemente, na forma das cidades.

Quando a vila invadiu a segunda dimensão, ela não se tornou uma cidade bidimensional. Isso só aconteceria se as casas preenchessem homogeneamente o espaço bidimensional, e esse não é o caso. Quando a vila começou a se expandir pela colina ela adquiriu uma dimensão espacial que é maior que 1 (ela não é mais construída apenas em torno de uma linha) e, ao mesmo tempo, menor que 2 (ela não preenche homogeneamente o espaço bidimensional). Ou seja, quando as casas começaram a ser construídas na colina, a vila passou a ter uma dimensão fracionária. Objetos físicos com dimensão não inteira, ou seja, que possuem dimensão fracionária, também são chamados de fractais (Mandelbrot, 1982). Na verdade, um fractal é qualquer coisa (por exemplo, objeto, fenômeno) que apresente propriedades 
similares em todas as suas escalas. No caso de um objeto físico fractal há um número que se preserva em todas as escalas de tamanho desse objeto, chamado de dimensão fractal. Saber esse número é interessante pois pode revelar algum tipo de padrão. De fato, cidades apresentam dimensões fractais, e esse número pode revelar propriedades universais no fenômeno urbano. No caso específico da Estação de Carrancas não é possível determinar sua dimensão fractal justamente por ela ser uma pequena vila. Na verdade, esse número só é computável quando olhamos para o sistema em questão sob diferentes escalas de tamanho. Neste sentido, só é possível determinar a dimensão fractal de cidades suficientemente grandes.

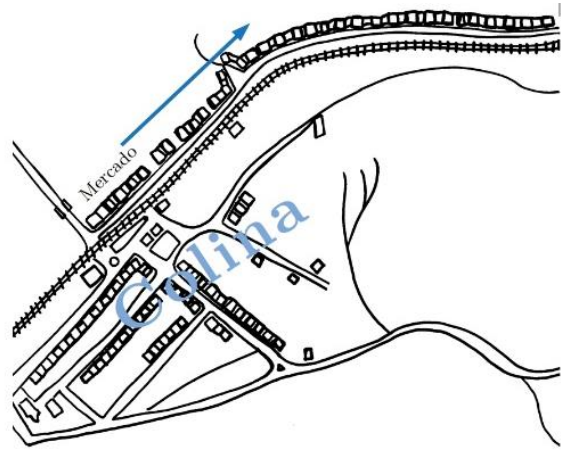

Figura 4. Imagem da Estação de Carrancas, uma pequena vila do interior de Minas Gerais. No início dos anos 70, a vila era formada por uma única rua (veja seta na figura) construída em torno de uma ferrovia, tendo um mercado em uma das extremidades. Nessa ocasião, ela era um exemplo de cidade unidimensional, no sentido de que ela se estendia ao longo de uma linha. Nos anos 80 a vila começa a se expandir sobre uma colina invadindo a segunda dimensão espacial (fonte: elaborada pelo autor).

Existem várias técnicas para se determinar a dimensão fractal de uma cidade. Uma delas é o método box counting, (Shen, 2012) que consiste em contar o número $n$ de quadrados de largura $s$ que cobrem toda a superfície urbanizada da cidade, conforme descrito na Figura (5a). A cidade será um fractal se essas duas quantidades se relacionarem por uma lei de potência do tipo descrito na Equação 1.

$$
n(s) \propto s^{-D_{f}}
$$

Se este modelo se ajusta bem aos dados então $D_{f}$ é a própria dimensão fractal da cidade. Uma forma de verificar se os dados são bem descritos por uma lei de potência é plotando- os em um gráfico log-log. Dados prescrevendo linha reta num gráfico log-log significam que o fenômeno é regido por uma lei de potência, como dada pela Eq. (1). Isto é, linha reta no gráfico log-log significa que estamos lidando com um fenômeno fractal. A figura (5b) mostra o gráfico (log-log) de $n(s)$ versus $s$ para a cidade de Nova York. Note um perfeito ajuste entre os dados e a lei de potência (representado pela linha reta). A inclinação da reta (expoente da lei de potência) nos dá a dimensão fractal da cidade de Nova York: $D_{f}=1.70 \pm 0.03$ (Shen, 2012). A aplicação dessa mesma metodologia para diferentes cidades pelo mundo permite construir um histograma de $D_{f}$, conforme apresentado na figura (6), no qual pode-se verificar que a dimensão das cidades flutua em torno de 1.7. O que significa, como já tínhamos apontado, que as cidades não são linhas retas nem preenchem homogeneamente o espaço bidimensional, o que justifica esse número fracionário da dimensão.

As cidades que estão na extrema esquerda do histograma (aquelas com dimensões fractais em torno de 1.3 e 1.4) são mais próximas da uni-dimensionalidade do que da bidimensionalidade. Por exemplo, a cidade de Tóquio, que possui $D_{f}=1.312$, foi construída em torno de um lago e acaba tendo uma distribuição espacial mais linear e, consequentemente, uma dimensão fractal menor se comparado com outras cidades.

Na próxima seção essas questões de escala continuarão a ser tratadas, mas agora considerando aspectos socioeconômicos e infraestruturais das cidades.

\section{Economia de Escala}

Podemos denominar por fractal não apenas objetos físicos, mas também qualquer quantidade/fenômeno que manifeste algum tipo de invariância de escala. Quando isso acontece, costuma-se dizer que essa quantidade ou fenômeno é livre de escala. Além disso, um fenômeno livre de escala geralmente pode ser representado por uma equação de potência, que é uma espécie de assinatura de um comportamento independente de escala (Newman, 2005). Por exemplo, evidências sugerem que certas métricas urbanas, que representaremos por $Y$, estão diretamente relacionadas com o número $N$ de habitantes de uma cidade a partir de 
uma lei de potência do tipo descrito na Equação 2.

a)

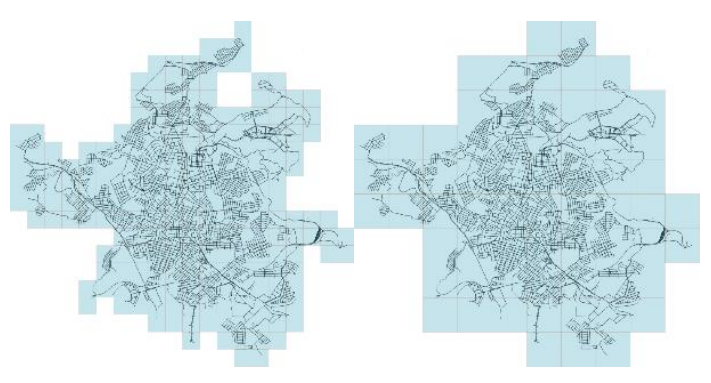

b)

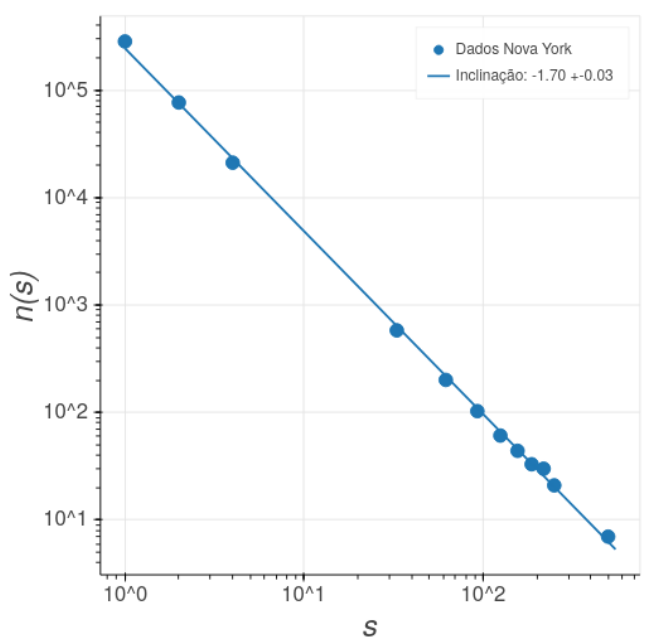

Figura 5. Exemplo ilustrativo do método boxcounting. a) Uma área urbanizada coberta por quadrados de dois tamanhos distintos. No

primeiro (à direita), com quadrados de lado $s=2$ (unidade arbitrária qualquer), são necessários $n(s=2)=66$ quadrados para cobrir toda a área urbanizada. Já no segundo, com quadrados de lado $s=1$, são necessários $n(s=1)=198$ quadrados para cobrir a mesma área urbanizada. b) Exemplo de aplicação do método: Gráfico (log-log) do número $n(s)$ de quadrados de lado $s$ necessários para cobrir toda a área urbana de Nova York, em função de $s$. Lei de potência (linha azul) se ajusta bem aos dados e, portanto, a inclinação da reta nos dá a dimensão fractal de Nova York: $D_{f}=1.70 \pm 0.03$ (fonte: elaborada pelo autor).

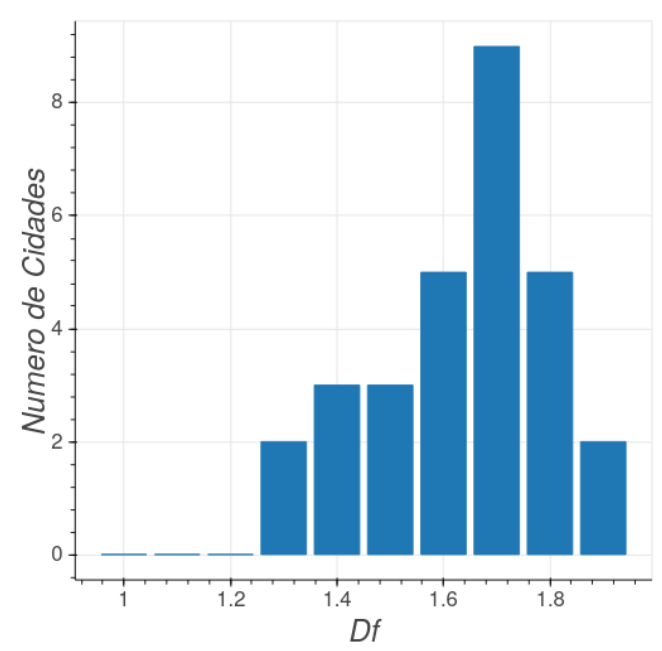

Figura 6. Histograma de $D_{f}$ para algumas cidades pelo mundo. Os valores da dimensão fractal estão distribuídos em torno de $D_{f}=1.7$. (fonte: adaptado de Shen, 2012; Ribeiro et al., 2017; Batty e Longley, 1994).

$$
Y=Y_{0} N^{\beta}
$$

Aqui, $Y_{0}$ e $\beta$ são parâmetros específicos de uma determinada métrica urbana (Ribeiro et al., 2017; Batty e Longley, 1994; Newman, 2005; Bettencourt, 2013). A figura (7) ilustra a boa descrição da equação acima aos dados empíricos quando $Y$ representa o PIB (produto interno bruto) para cidades brasileiras e norte-americanas. Isso sugere que o PIB é regido por propriedades livres de escala, isto é, os sistemas urbanos apresentam comportamentos similares em todas as escalas de tamanho. Isso é exatamente um comportamento fractal, apesar de estarmos lidando com uma variável econômica e a população, e não um objeto físico e um comprimento. Apesar da grande complexidade que está por trás dos fenômenos urbanos, no final das contas a relação entre PIB e a população parece obedecer uma relação relativamente simples, dada pela equação (2).

Evidências empíricas mostram que o valor assumido por $\beta$, o expoente de escala, determina o tipo/categoria de variável urbana. Variáveis socioeconômicas, como o PIB, salários, número de caso de doenças socio-transmissíveis, etc., apresentam $\beta>1$. Este é o chamado regime superlinear, no sentido que quando duplicamos o tamanho da cidade, aumenta-se a quantidade per capita destas variáveis. Por exemplo, a quantidade 
per capita destas variáveis socioeconômicas tende a aumentar em 16\%, em média, quando se dobra o tamanho de uma cidade - o chamado increasing return to scale (rendimentos crescentes em função da escala) em cidades maiores.

A explicação mais aceita para essa propriedade superlinear tem a ver com a interatividade das pessoas, que também cresce superlinearmente com o tamanho da cidade. Por exemplo, número de chamadas e contatos telefônicos crescem superlinearmente com o tamanho da população (Schläpfer et al., 2014; Stier, Berman e Bettencourt, 2020). Dessa forma, a interatividade é apontada como o grande potencializador da criatividade e da produtividade revertendo em benefícios sociais e econômicos. Podemos dizer que, quanto maior é a cidade, mais interação e contatos entre pessoas ela proporciona, gerando cidades mais ricas e criativas.

Porém há uma consequência negativa da maior conectividade entre pessoas em cidades maiores. Cidades grandes são mais susceptíveis a epidemias, como estamos observando no caso da Covid-19 (Stier, Berman e Bettencourt, 2020). Resultados análogos também aparecem em outras doenças sócio-transmissíveis, como é o caso da AIDS, que também cresce de forma superlinear com o tamanho da cidade (Bettencourt et al., 2007).

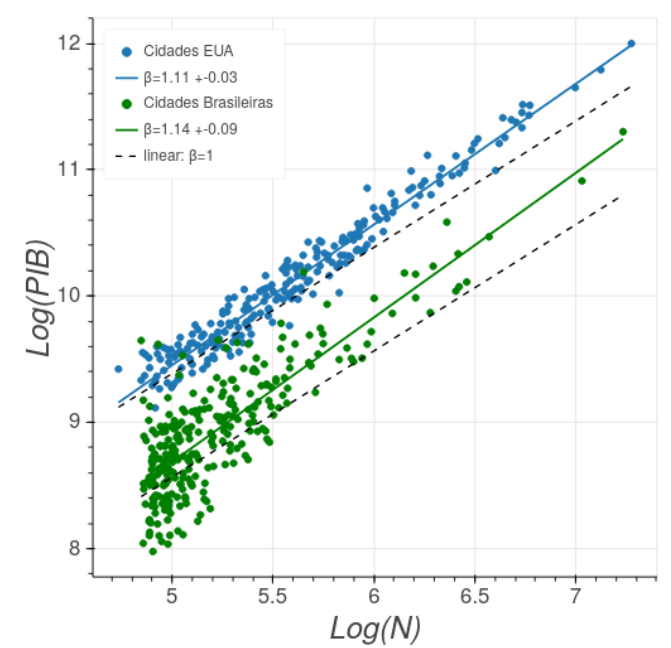

Figura 7. Gráfico (log-log) do PIB em função da população $N$, para as cidades brasileiras e norte americanas. As linhas cheias (azul e verde) são aquelas que melhor se ajustam à tendência dos pontos, e revelam propriedade tipo lei de potência. Os dois países apresentam expoentes de escala $\beta>1$ (superlinear), com valores muito próximos entre si, apesar da diferença socioeconômica entre esses dois países. As linhas tracejadas representam a situação linear $(\beta=1)$, e são apresentadas apenas para enfatizar o comportamento superlinear da tendência dos dados (fonte: elaborada pelo autor).

Em contrapartida, outros tipos de variáveis geralmente associadas à infraestrutura e estrutura urbana apresentam $\beta<1$.

Exemplos dessas variáveis são o número de hospitais, número de escolas, número de postos de gasolina, área urbanizada, comprimento total de ruas, comprimento de cabos elétricos, etc. Este é o chamado regime sublinear, no sentido que quando se aumenta o tamanho da cidade, diminui-se o número per capita destas quantidades. Há, neste caso, uma economia de infraestrutura em cidades maiores: cidades maiores fazem mais com menos. Por exemplo, quando aumentamos o tamanho da cidade a quantidade de postos de gasolina por habitante tende a diminuir. Essencialmente, a quantidade per capita dessas variáveis de infraestrutura reduzem em $16 \%$, em média, quando se dobra o tamanho de uma cidade.

Finalmente, tem-se um terceiro tipo de variável, que apresenta $\beta \approx 1$. Este é o regime linear, dado por variáveis que estão relacionadas com necessidades individuais, tais como consumo de água, número de empregos e número de casas alugadas.

Uma importante propriedade da lei de escala em sistemas urbanos é que os dados empíricos mostram características universais nessas variáveis. Por exemplo, Brasil e os Estados Unidos apresentam PIB com o expoente de escala $\beta$ muito parecidos (1.14 e 1.11 , respectivamente), apesar desses países serem completamente diferentes em termos econômicos e sociais (Bettencourt, 2013; Schläpfer et al., 2014; Stier, Berman e Bettencourt, 2020; Meirelles et al., 2020). Evidências empíricas em outros países pelo mundo têm mostrado o mesmo comportamento de variáveis socioeconômicas como o PIB, apresentando $\beta \approx 1.16$ (superlinear), enquanto variáveis de infraestrutura têm apresentado $\beta \approx 0.84$ (sublinear). Essa universalidade sugere que mesmo com todas as diferenças culturais, históricas e geográficas entre os países, aparentemente certas métricas urbanas são regidas por propriedades universais e uma lei de potência simples, com expoentes de escala bem definidos. A percepção dessa aparente 
universalidade pode ser um forte indício de que é possível identificar leis e quem sabe até propor teorias mais abrangentes para explicar o fenômeno urbano.

\section{Tycho Brahe, Kepler e Newton}

No final do séc. XVI, Tycho Brahe tinha a mais notável coleção de dados celestes, o que incluía, por exemplo, a trajetória de todos os planetas visíveis da época. Mesmo nos padrões que temos hoje, podemos dizer que ele tinha um Big Data disponível, devido ao tamanho do banco de dados e também pelo fato deles estarem sendo constantemente atualizados. Apesar disso, Tycho Brahe só tinha uma coleção de números, sem qualquer teoria para explicá-los ou descrevê-los. Tycho Brahe representa a primeira fase do desenvolvimento da mecânica celeste: a fase da coleta dos dados. Essa fase será chamada simplesmente de fase Tycho Brahe.

Então veio Kepler, que começou a trabalhar com Tycho Brahe em 1600 e, posteriormente, com os seus dados astronômicos. Kepler percebeu alguns padrões nos dados que tinha em mãos, hoje conhecidas como as "três leis de Kepler". São elas: i) lei das órbitas elípticas $^{2}$; ii) lei das áreas ${ }^{3}$; e iii) lei dos períodos ${ }^{4}$. Com a identificação destas leis, Kepler respondeu como os planetas se movem, mesmo sem ele saber dizer por que eles movem. Neste sentido, Kepler representa a segunda fase do desenvolvimento da mecânica celeste: a fase da identificação de padrões. Essa fase será chamada simplesmente de fase Kepler.

Nesse contexto, entra em cena Newton com as três leis da mecânica: i) lei da inércia; ii) relação entre força, aceleração e massa, i.e. $F=m a$; e iii) lei da ação e reação. E, finalmente, a lei da gravitação: $F=G m_{1} m_{2} /$ $r^{2}$, que diz que matéria atrai matéria numa relação inversa com o quadrado da distância. Com estas leis ele identifica que a terra e o céu são regidos pelos mesmos princípios. Isto é, a partir de um mesmo conjunto de regras ele explica tanto o movimento dos corpos aqui na Terra, quanto o movimento dos planetas ao redor do sol. Com suas leis, Newton mostrou porque os corpos se movem 5 , e suas descobertas representam a terceira fase do desenvolvimento da mecânica celeste: a fase da explicação dos fenômenos. Essa fase será chamada simplesmente de fase Newton.
Acredito que no contexto da ciência das cidades estamos na fase Kepler. Quero dizer, já superamos o período Tycho Brahe no contexto urbano, no sentido de que já possuímos uma enorme quantidade de dados disponíveis, como posição e movimento das pessoas em tempo real, fluxos diários inter e intra-cidades, e muito mais. E já identificamos alguns padrões nestes dados como, por exemplo, as propriedades de escala que foram discutidas anteriormente. Contudo existem muitos outros padrões já identificados, como os que se referem à mobilidade urbana (Alessandretti et al., 2018), abundância de categorias de empresas (Strumsky et al., 2016), e a própria dimensão fractal das cidades (Murcio et al., 2015). Na verdade, estamos numa época de tamanha sofisticação técnica que podemos jogar os dados em um supercomputador e deixar que os próprios algoritmos estatísticos encontrem por eles mesmos os padrões entre as métricas urbanas (Anderson, 2008).

Mas seria possível atingir a fase Newtoniana da ciência das cidades? Em outras palavras, será possível organizar todos os dados que possuímos e todos os padrões já identificados em um conjunto de equações compactas e inteligíveis que nos permitam explicar os dados e fazer previsões? Isso foi feito de forma bastante elegante para a mecânica, há pelo menos 300 anos. Será isso possível também para o fenômeno urbano? Ou seria esse um sonho impossível de ser alcançado, dada a complexidade e o número imensurável de variáveis envolvidas no contexto urbano?

\section{Uma nova ciência das cidades ou uma nova ciência para as cidades?}

Mas seria realmente necessário atingir a fase newtoniana das cidades? Não seria suficiente deixarmos os próprios algoritmos e os números falarem por si só? Por exemplo, o Google usa seus algoritmos para dizer que uma webpage é melhor que outra, mesmo não sabendo exatamente por que ela é melhor, mas simplesmente porque o algoritmo está dizendo que é, e isso é o bastante. Porém, encerrar-se neste tipo de estratégia seria condenar exatamente o que entendemos por ciência. Ou seria a ciência que conhecemos construída apenas para aqueles problemas e fenômenos que podem ser teorizados de alguma forma? O que não se aplicaria a fenômenos compostos por um 
número imensurável de variáveis interconectadas, como é o caso das cidades.

Fica então a dúvida: estaríamos atrás de uma ciência das cidades, nos mesmos moldes das disciplinas bem estabelecidas? Neste caso, teríamos que seguir os mesmos ritos da ciência tradicional. Esta ciência tradicional funcionou muito bem nos últimos séculos em questões que envolvem um número pequeno de variáveis envolvidas; tais como um bloco deslizando em uma superfície de gelo, ou um corpo suficientemente pesado caindo livremente. Esses tipos de problemas permitem ser equacionados e descritos por regras simples e compactas, justamente por se tratarem de problemas com um número finito de variáveis essenciais (Weaver, 1948). Mas, e quando temos sistemas complexos como as cidades? As cidades são sistemas que se auto-organizam, o que talvez as tornem impossíveis de serem descritas ou explicados por regras simples. Dessa forma,

\section{Notas}

${ }^{1}$ https://nacoesunidas.org/onu-mais-de-70-dapopulacao-mundial-vivera-em-cidades-ate-2050/

${ }^{2}$ Os planetas descrevem orbitas elípticas, com o sol ocupando um dos focos.

${ }^{3}$ A linha que liga o planeta ao Sol varre áreas iguais em tempos iguais.

\section{Referências}

Alessandretti, L., Sapiezynski, P., Sekara, V., Lehmann, S., \& Baronchelli, A. (2018) Evidence for a conserved quantity in human mobility. Nature Human Behaviour, 2 (7), 485-491.

Anderson, C. (2008) The end of theory: The data deluge makes the scientific method obsolete. Wired magazine, 16 (7), 1-03.

Asprone, D., Cavallaro, M., Latora, V., Manfredi, G., \& Nicosia, V. (2013).

Assessment of urban ecosystem resilience using the efficiency of hybrid social-physical complex networks. Arxiv. [Preprint] Disponível em: https://arxiv.org/abs/ 1302.3263. [Consultado em: 30 junho 2020].

Bak, P. (1999) How nature works: the science of self-organized criticality. New York, Copernicus.

Ball, P. (2004) Critical mass: how one thing leads to another. New York, Farrar, Straus and Giroux. talvez estaríamos atrás de uma ciência realmente nova, uma ciência baseada puramente nos dados, que abandonaria o conceito de teoria. Uma ciência feita exclusivamente para tratar o fenômeno urbano: uma ciência para as cidades.

\section{Agradecimentos}

Gostaria de agradecer meu caro amigo Vinicius M. Netto, não só pela nossa intensa parceria científica, mas também pelo incentivo inicial para eu escrever este artigo. Gostaria de agradecer também Romulo Krafta pela leitura prévia do manuscrito e pelas valorosas sugestões. Este trabalho só foi possível pela ajuda inestimável de meu estudante Victor Cabral e de minha esposa Josiane O.P. Ribeiro com as figuras aqui presentes. Quero agradecer também o apoio financeiro do Cnpq (405921/2016-0) e da Capes (88881.119533/2016-01).

\footnotetext{
${ }^{4}$ Os quadrados dos tempos de translação dos planetas são iguais ao cubo dos semi-eixos maiores das órbitas.

${ }^{5}$ Apesar de que Einstein, mais tarde, com a teoria da relatividade, deu outra explicação para o movimento dos corpos: a distorção do espaço tempo.
}

Barthelemy, M. (2019) The statistical physics of cities. Nature Reviews Physics, 1 (6), 406415.

Batty, M. (1971) Modelling cities as dynamic systems. Nature, 231 (5303), 425-428.

Batty, M. (2013) The new science of cities. Cambridge, MIT press.

Batty, M., Longley, P. A. (1994) Fractal cities: a geometry of form and function. Cambridge, Academic press.

Bettencourt, L. M. (2013). The origins of scaling in cities. Science, 340 (6139), 14381441.

Bettencourt, L. M., Lobo, J., Helbing, D., Kühnert, C., West, G. B. (2007) Growth, innovation, scaling, and the pace of life in cities. Proceedings of the national academy of sciences, 104 (17), 7301-7306.

Birkin, M., Clarke, G. P. (1991) Spatial interaction in geography. Geography Review, $4(5), 16-24$ 
Boccara, N. (2010) Modeling complex systems. New York, Springer Science \& Business Media.

Haff, P. K. (2000) Rivers, blood and transportation networks. Nature, 408 (6809), 159-160.

Louf, R., Roth, C., \& Barthelemy, M. (2014) Scaling in Transportation Networks. PLoS ONE, 9 (7), 1-8.

Mandelbrot, B. B. (1982) The Fractal Geometry of Nature. San Francisco, W. H. Freeman and Co.

Meirelles, J., Neto, C. R., Ferreira, F. F., Ribeiro, F. L., \& Binder, C. R. (2018) Evolution of urban scaling: Evidence from Brazil. PLoS ONE, 13 (10), e0204574.

Mitchell, M. (2009). Complexity: A guided tour. New York, Oxford University Press.

Murcio, R., Masucci, A. P., Arcaute, E., \& Batty, M. (2015) Multifractal to monofractal evolution of the London street network. Physical Review E, 92 (6), 1-12.

Newman, M. E. (2005) Power laws, Pareto distributions and Zipf's law. Contemporary physics, 46 (5), 323-351.

Peter W. (2005) Cities of the world: a History in maps. Los Angeles, University of California Press.

Portugali, J. (2000) Self-Organization and the city. New York, Springer.
Ribeiro, F. L., Meirelles, J., Ferreira, F. F., Neto, C. R. (2017) A model of urban scaling laws based on distance dependent interactions. Royal Society open science, 4 (3), 160926.

Schläpfer, M., Bettencourt, L. M., Grauwin, S., Raschke, M., Claxton, R., Smoreda, Z., ... \& Ratti, C. (2014) The scaling of human interactions with city size. Journal of the Royal Society Interface, 11 (98), 20130789.

Shen, G. (2002). Fractal dimension and fractal growth of urbanized areas. International Journal of Geographical Information Science, 16 (5), 419-437.

Stier, A., Berman, M., \& Bettencourt, L. (2020) COVID-19 attack rate increases with city size. Mansueto Institute for Urban Innovation Research Paper Forthcoming, 19. Disponível em SSRN:

https://ssrn.com/abstract=3564464

Strano, E., Nicosia, V., Latora, V., Porta, S., \& Barthélemy, M. (2012) Elementary processes governing the evolution of road networks. Scientific reports, 2, 1-8.

W. Weaver (1948) Science and complexity, American scientist, 36(4), 536-544.

Youn, H., Bettencourt, L. M., Lobo, J., Strumsky, D., Samaniego, H., \& West, G. B. (2016) Scaling and universality in urban economic diversification. Journal of The Royal Society Interface, 13(114), 20150937. 